Acta Crystallographica Section D

\section{Biological \\ Crystallography}

ISSN 0907-4449

\section{Gordon Joyce, Sergei Radaev and Peter D. Sun*}

Structural Immunology Section, Laboratory of Immunogenetics, National Institute of Allergy and Infectious Diseases, National Institutes of Health, 12441 Parklawn Drive, Rockville, Maryland 20852, USA

Correspondence e-mail: psun@nih.gov

\title{
A rational approach to heavy-atom derivative screening
}

Despite the development in recent times of a range of techniques for phasing macromolecules, the conventional heavy-atom derivatization method still plays a significant role in protein structure determination. However, this method has become less popular in modern high-throughput oriented crystallography, mostly owing to its trial-and-error nature, which often results in lengthy empirical searches requiring large numbers of well diffracting crystals. In addition, the phasing power of heavy-atom derivatives is often compromised by lack of isomorphism or even loss of diffraction. In order to overcome the difficulties associated with the 'classical' heavy-atom derivatization procedure, an attempt has been made to develop a rational crystal-free heavy-atom derivative-screening method and a quick-soak derivatization procedure which allows heavy-atom compound identification. The method includes three basic steps: (i) the selection of likely reactive compounds for a given protein and specific crystallization conditions based on pre-defined heavy-atom compound reactivity profiles, (ii) screening of the chosen heavy-atom compounds for their ability to form protein adducts using mass spectrometry and (iii) derivatization of crystals with selected heavy-metal compounds using the quicksoak method to maximize diffraction quality and minimize non-isomorphism. Overall, this system streamlines the process of heavy-atom compound identification and minimizes the problem of non-isomorphism in phasing.

\section{Introduction}

The use of heavy-atom phasing still remains a major technique in de novo macromolecular crystal structure determination. However, there are a number of difficulties associated with the technique which have limited its widespread use in recent years. The traditional method usually entails the soaking of multiple crystals in numerous heavy-atom compound solutions for days to weeks (Blundell \& Johnson, 1976). The success of a derivatization is then evaluated through X-ray diffraction data analysis. While the method has been highly utilized in the past, it is too inefficient to support the demands of modern crystallography. The obvious difficulties in the conventional heavy-atom derivative-screening process are that (i) it is an empirical hit-or-miss process based on random screening of numerous heavy-atom compounds, (ii) it requires multiple crystals and (iii) it is a lengthy process requiring multiple X-ray data acquisitions and analyses. The expectations of high-throughput structure determination demand a new, rapid and rational heavy-atom screening procedure. Additionally, the ever-increasing application of crystallo-
Received 11 March 2009 Accepted 9 December 2009 
Table 1

List of the most reactive compounds for heavy-atom derivatization of proteins.

\begin{tabular}{ll}
\hline Ranking of the most reactive compounds & Derivatization (\%) \\
\hline Ethylmercury(II) phosphate & 69.4 \\
Methylmercury(II) acetate & 66.6 \\
Sodium tetrachloroaurate & 61.1 \\
Potassium tetrabromoplatinate & 55.5 \\
Potassium tetrachloroaurate & 52.7 \\
Ammonium tetrachloroplatinate & 50.0 \\
Gold(III) chloride & 47.2 \\
Diaminoplatinum dinitrate & 47.2 \\
Thiomersal & 47.2 \\
Mercury(II) acetate & 47.2 \\
PCMBS & 47.2 \\
Potassium tetrachloroplatinate & 44.4 \\
Potassium tetranitroplatinate & 44.4 \\
Lead acetate & 43.3 \\
Potassium hexabromoplatinate & 41.7 \\
Methylmercury(II) chloride & 38.8 \\
Mersalyl & 38.8 \\
Mercury(II) bromide & 36.1 \\
Mercury(II) cyanide & 33.3 \\
Gold chloride & 33.3 \\
Platinum potassium thiocyanate & 33.3 \\
Lead nitrate & 33.3 \\
\hline
\end{tabular}

graphy to difficult projects with often limited amounts of protein samples and crystals make the lengthy routine screening of heavy-atom derivatives impractical.

Here, we summarize the development of a rapid rational procedure for the identification of heavy-atom compounds for phasing. Specifically, we have developed an approach to enable the selection of heavy-atom compounds based on known reactivities in specific crystallization conditions (Agniswamy et al., 2008). Mass spectrometry is then used to provide a reliable, rapid and crystal-free method for assessing the likely heavy-atom compounds for derivatization (Sun \& Hammer, 2000). A quick-soak method is then used to minimize non-isomorphism and maximize the phasing power of heavy-atom derivatives (Sun et al., 2002; Sun \& Radaev, 2002).

\section{Selection of reactive heavy-atom compounds based on their reactivity profiles}

The heavy-metal compounds used in crystallography are generally classified as either class A or class B (Blundell \& Johnson, 1976; Blundell \& Jenkins, 1977). Class A heavy-metal compounds, such as the lanthanides and actinides (primarily uranium), tend to bind to electronegative protein ligands through charge interactions, e.g. $\mathrm{UO}_{2}^{2+}$ binds to the carboxylate group of glutamate and aspartate, as seen in the heavyatom-bound insulin structure (Blundell et al., 1971) and also in the prealbumin structure (Blake et al., 1974). In contrast, class B metals such as platinum, gold and mercury bind covalently to reactive amines and sulfhydryl groups (Islam et al., 1998; Rould, 1997). However, other class B metals such as lead and thallium show a different reactivity and tend to interact with hydroxyl groups. Successful heavy-atom derivatization depends not only on the availability of specific amino-acid ligands in a given protein but also to a great extent on the
Table 2

Summary of peptide derivatization.

The numbers given are for highly reactive compounds which gave greater than $50 \%$ derivatization in a single reaction.

\begin{tabular}{llrrlll}
\hline & \multicolumn{7}{l}{ Peptides } \\
\cline { 2 - 7 } & Met & His & Cys & Asp/Asn/Glu/Gln & Tyr & Total \\
\hline Sodium acetate & 7 & 4 & 9 & 2 & 3 & 25 \\
Sodium cacodylate & 6 & 3 & 11 & 1 & 3 & 24 \\
Sodium citrate & 7 & 0 & 8 & 0 & 0 & 15 \\
MES & 8 & 26 & 19 & 6 & 4 & 63 \\
HEPES & 7 & 7 & 16 & 5 & 3 & 38 \\
Tris & 4 & 3 & 9 & 2 & 2 & 20 \\
Pt compounds & 6 & 11 & 3 & & & \\
Hg compounds & 2 & 7 & 10 & & & \\
Au compouds & 2 & 4 & 3 & & & \\
\hline
\end{tabular}

crystallization conditions. Buffer and $\mathrm{pH}$ are known to affect the reactivity and solubility of heavy-atom compounds both through chelating heavy atoms and influencing the protonation state of the reactive groups.

To systematically assess the effect of buffer on heavy-atom reactivities, we carried out a series of derivatization experiments using peptides with a single reactive residue (e.g. the methionine-containing peptide GEAGMASAGGAG) and class B heavy-metal compounds. These heavy-atom compounds generally form covalent adducts with amino-acid ligands and their reactivity depends less on the tertiary conformation of the ligands. Peptides with a single cysteine, methionine or histidine residue were assessed for reactivity with platinum, gold and mercury compounds, while peptides containing a single aspartate, glutamate, asparagine, glutamine or tyrosine residue were used in derivatization experiments with lead-containing compounds. A total of 43 heavy-atom compounds were tested for peptide reactivity in 12 buffer conditions over a wide range of $\mathrm{pH}$. The results are tabulated in Agniswamy et al. (2008) and can be found at http:// sis.niaid.nih.gov/cgi-bin/heavyatom_reactivity.cgi. The database can be used to select compounds that are likely to derivatize a given protein of interest under selected buffer conditions.

As expected, heavy-metal compound reactivities depend strongly on buffer and $\mathrm{pH}$ conditions. Overall, MES and citrate buffers are the most and least supportive for heavyatom derivatization experiments, respectively (Table 1). Therefore, proteins crystallized under MES buffer conditions are likely to be derivatized by a larger range of compounds than those crystallized in any other buffer. Among the basic $\mathrm{pH}$ buffers, reactions carried out in HEPES buffer have a greater success rate than those carried out in Tris buffers. However, depending on the peptide ligands available, heavy atoms may react preferentially in either HEPES or Tris buffer. The $\mathrm{pH}$ preference of heavy-metal reactivity is also apparent from this study. Gold potassium bromide, potassium tetrabromoaurate, gold potassium thiocyanide and trimethyllead acetate (TMLA) all show high levels of derivatization at slightly acidic to basic $\mathrm{pH}$ values, while potassium tetracyanoplatinate, gold sodium thiosulfate, mercury(II) chloride, methylmercury(II) bromide, $p$-chloromercuric benzoic acid, 
dichloroethylenediaminoplatinate and potassium hexachloroplatinate all react strongly under acidic conditions. It is interesting that $\mathrm{K}_{2} \mathrm{IrCl}_{6}$ and $\mathrm{K}_{2} \mathrm{OsCl}_{6}$ are observed to react consistently with the Met, Cys and His peptides in the vast majority of conditions examined, but the percentage of total peptide in a reaction which forms a heavy-atom adduct is consistently lower than that seen for other heavy-atom compounds.

Another observation which is clear from the data is that a number of compounds are highly reactive over a broad range of buffer and $\mathrm{pH}$. The 22 most reactive compounds are listed in Table 1 and they include the seven compounds that were previously identified as highly successful in protein-derivatization experiments (Garman \& Murray, 2003; Boggon \& Shapiro, 2000). Other results that stand out include the observation that Met and Cys can be derivatized by at least four heavy-atom compounds in all buffers (Table 2). Methionine and histidine residues are the most reactive with platinum compounds, while cysteine preferentially reacts with mercury compounds. Thus, for proteins rich in methionine and histidine platinum compounds should be the first choice for

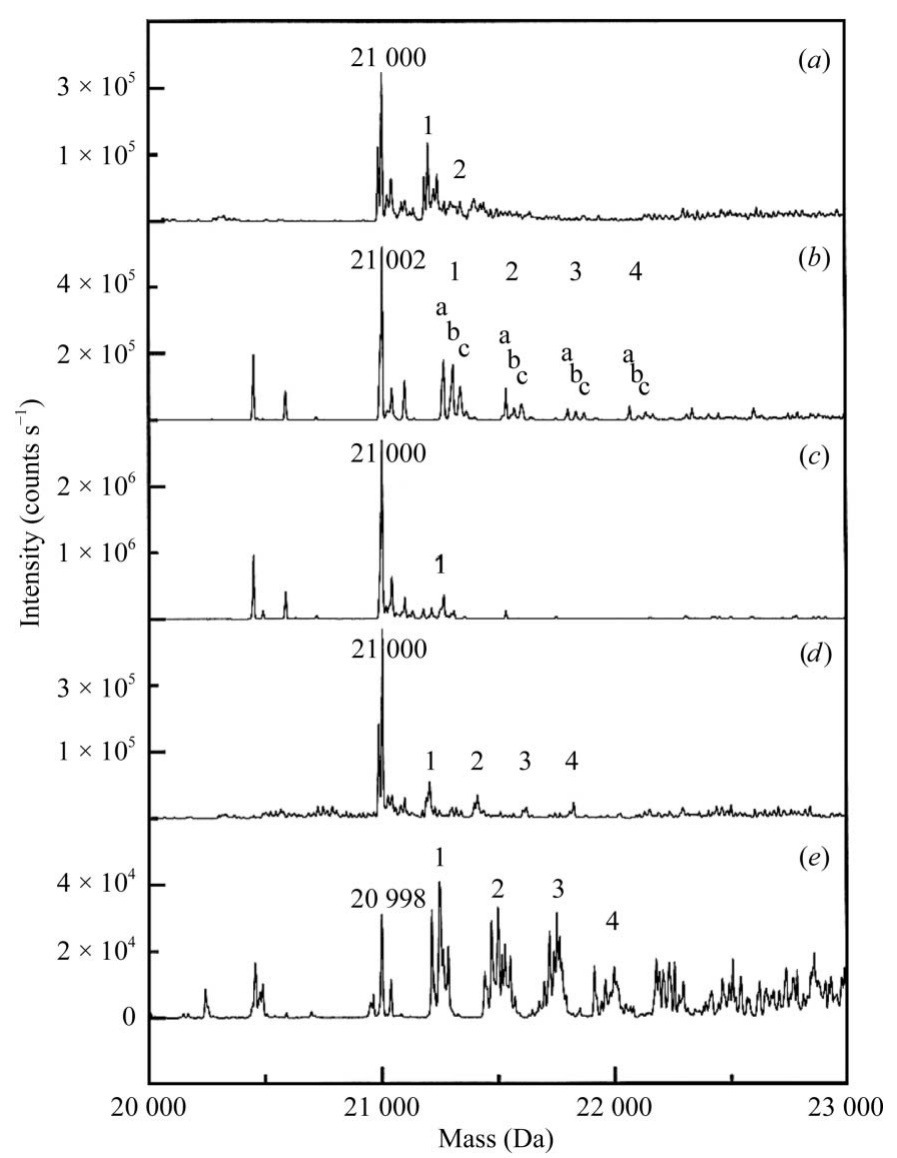

Figure 1

Mass-spectrometric profiles of Fc $\gamma$ RIII (calculated molecular weight $20996 \mathrm{Da}$ ) reacted with (a) $\mathrm{HgCl}_{2}$, (b) $\mathrm{K}_{2} \mathrm{PtCl}_{4}$, (c) TMLA, (d) lead acetate or $(e) \mathrm{KAu}(\mathrm{CN})_{2}$. The molecular weight of the residual native peak is labeled in each panel. The number of heavy atoms covalently attached to the protein is indicated above the adduct peaks (taken from Sun \& Hammer, 2000). screening, while mercury and gold compounds become the obvious candidates for proteins rich in free cysteines. Most importantly, the pH-dependent and buffer-dependent heavyatom reactivity profiles enable the user to avoid experiments with compounds that are nonreactive in specific buffers, even in an ideal experimental scenario such as the heavy-atom peptide experiment carried out here.

\section{Assessment of protein heavy-atom derivatization using mass spectrometry}

To replace the traditional time-consuming heavy-atom screening procedure, we utilized mass spectrometry for heavyatom derivative screening. This method not only enables rapid selection and optimization of the potential derivatives, but also eliminates the use of crystals, allowing streamlining of the heavy-atom derivatization process. Here, we present two test cases to illustrate the general applicability of this method.

\subsection{Derivatization of Fc $\gamma$ RIII}

The extracellular ligand-binding domain of the type III human Fc receptor, Fc $\gamma$ RIII, contains two immunoglobulinlike (Ig-like) domains with a molecular weight of $21000 \mathrm{Da}$ as measured by electrospray ionization mass spectrometry (ESI-MS). The derivatization reactions were prepared by mixing $0.5-1 \mu \mathrm{l}$ pre-dissolved heavy-atom compound solutions at various concentrations with $5-10 \mu \mathrm{Fc} \gamma \mathrm{RIII}$ at $2-5 \mathrm{mg} \mathrm{ml}^{-1}$ in water for $30 \mathrm{~min}$ at room temperature before infusion of the sample into the mass spectrometer. Two adducts of $\mathrm{HgCl}_{2}$ with molecular weights of 21198 and 21398 Da that corresponded to the addition of one and two $\mathrm{Hg}^{2+}$ ions, respectively, were

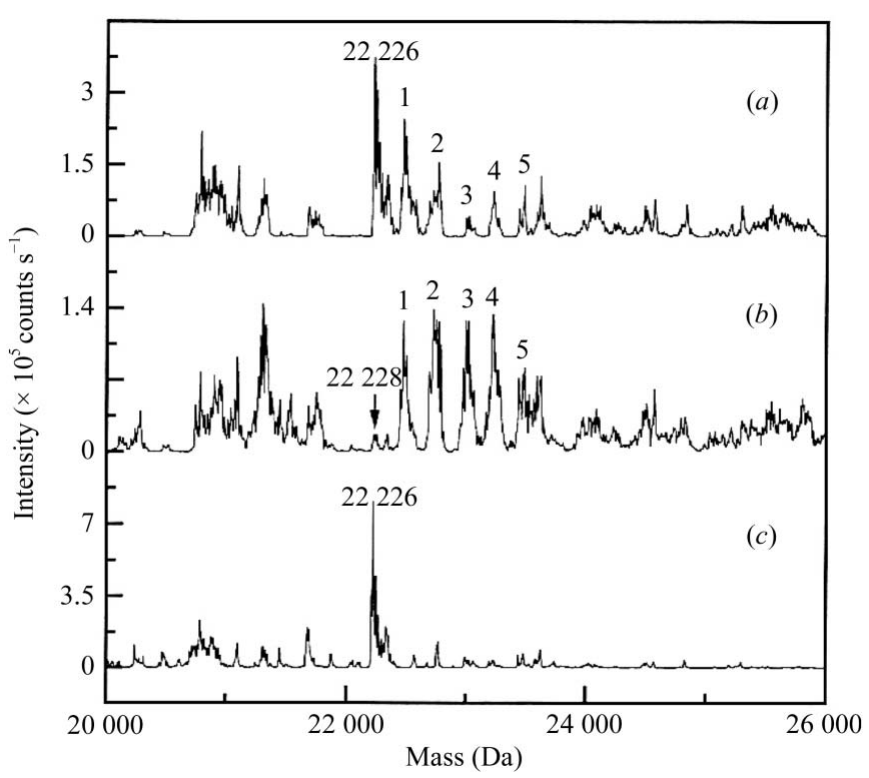

Figure 2

ESI-MS results for gold cyanide-derivatized KIR2DL2. The $\mathrm{KAu}(\mathrm{CN})_{2^{-}}$ derivatization reaction was carried out using heavy atom:protein molar ratios of $(a)$ 9:1 and $(b)$ 28:1, respectively. The $\mathrm{KAu}(\mathrm{CN})_{2}$-derivatized peaks are labeled 1-5. (c) Native KIR2DL2 has a molecular weight of 22226.0 Da (taken from Sun \& Hammer, 2000). 
detected in addition to the native peak (Fig. 1). Additionally, Fc $\gamma$ RIII was also found to react with $\mathrm{K}_{2} \mathrm{PtCl}_{4}$, TMLA, lead acetate and $\mathrm{KAu}(\mathrm{CN})_{2}$ (Fig. 1). Furthermore, the numbers of heavy-atom sites found by ESI-MS largely correlated with those found from crystallographic heavy-atom refinement (Sun \& Hammer, 2000).

\subsection{Derivatization of KIR2DL2}

The extracellular ligand-binding region of KIR2DL2 contains two Ig-like domains with a calculated molecular weight of $22228 \mathrm{Da}$. The crystal structure of the soluble receptor has previously been determined using $\mathrm{KAu}(\mathrm{CN})_{2}$ as the heavyatom phasing derivative (Snyder et al., 1999). Two reactions with molar $\mathrm{KAu}(\mathrm{CN})_{2}: \mathrm{KIR} 2 \mathrm{DL} 2$ concentration ratios of 9:1

Table 3

Derivatization conditions and phasing statistics of lysozyme derivatives (adapted from Sun et al., 2002).

\begin{tabular}{lllllllll}
\hline & $\mathrm{KAuCl}_{4}$ & \multicolumn{7}{c}{$\mathrm{K}_{2} \mathrm{PtCl}_{6}$} \\
\hline Derivatization $\dagger$ & $10 \mathrm{~m} M$, & $10 \mathrm{~m} M$, & $1 \mathrm{~m} M$, & $10 \mathrm{~m} M$, & $12 \mathrm{~m} M$, & $1 \mathrm{~m} M$, & $1 \mathrm{~m} M$, & $10 \mathrm{~m} M$, \\
& $10 \mathrm{~min}$ & $24 \mathrm{~h}$ & $48 \mathrm{~h}$ & $10 \mathrm{~min}$ & $10 \mathrm{~min}$ & $10 \mathrm{~min}$ & $22 \mathrm{~h}$ & $48 \mathrm{~h}$ \\
$R_{\text {iso }}$ & 0.201 & 0.462 & 0.349 & 0.176 & 0.208 & 0.111 & 0.087 & 0.213 \\
Heavy-atom peak height $\neq$ & & & & & & & \\
$\quad$ Site 1 $(\sigma)$ & 21.6 & $<4.0$ & 15.7 & 19.3 & 18.2 & $<5.0$ & 15.0 & 6.2 \\
Site 2 $(\sigma)$ & 12.8 & $<4.0$ & 9.3 & 16.3 & 16.5 & $<5.0$ & 10.5 & 11.0 \\
Site 3 $(\sigma)$ & 9.7 & $<4.0$ & & & & & & \\
\hline
\end{tabular}

$\dagger$ Heavy-atom soaking concentration, soaking time. $\ddagger$ The heavy-atom sites are shown as peak heights in standard deviations from the difference Fourier $\left(F_{\mathrm{PH}}-F_{\mathrm{P}}\right)$ map. For the $\mathrm{KAuCl}_{4}$ derivative the coordinates of sites 1,2 and 3 are $(-11.36,11.72,19.21),(-8.49,10.2,14.25)$ and $(3.30,7.94,9.84) \AA$, respectively. For the $\mathrm{K}_{2} \mathrm{PtCl}_{6}$ derivative the coordinates of site 1 and 2 are $(-10.957,10.957,9.23)$ and $(6.143,3.859,29.992) \AA$, respectively.

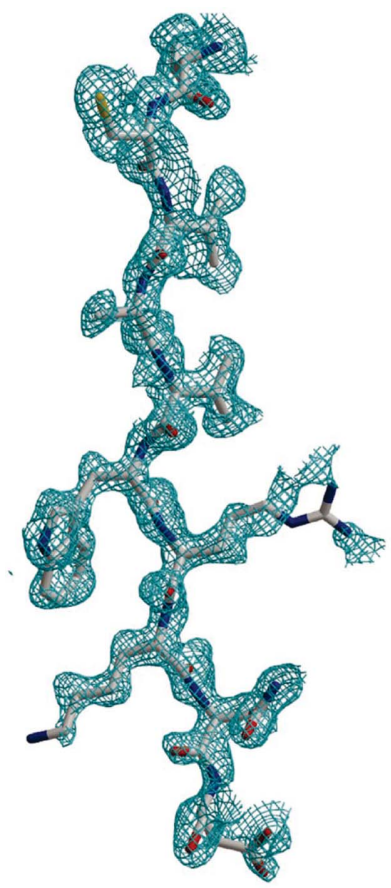

(a)

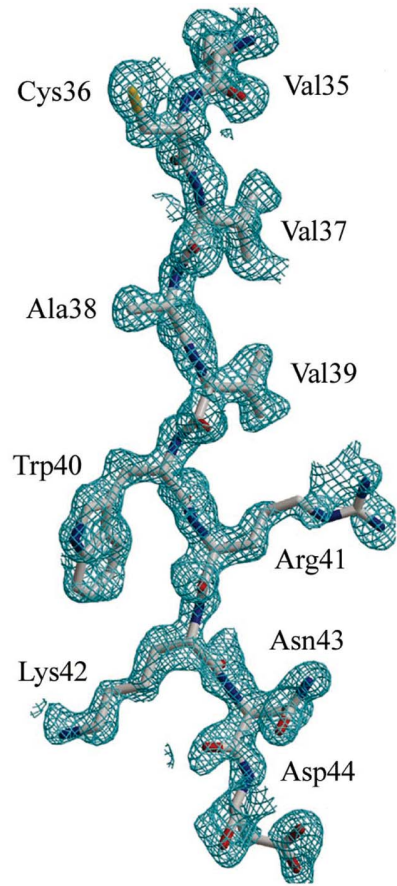

(b)

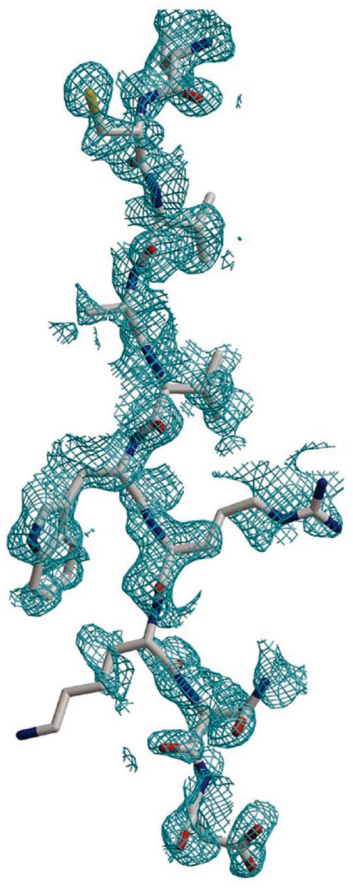

(c)
Figure 3

Experimental electron-density maps of $\mathrm{T} \beta \mathrm{RII}$ phased with $\mathrm{HgCl}_{2}$ derivatives. (a) A region of the MAD-phased electron-density map contoured at $1 \sigma$ with the corresponding refined model. $(b)$ SIRAS map produced by a 10 min quick-soak. (c) SIRAS map resulting from the long $12 \mathrm{~h}$ soak (taken from Sun \& Radaev, 2002). and 28:1, respectively, were carried out in solution for $30 \mathrm{~min}$ $6.5 \mu \mathrm{g}$ KIR2DL2 in each reaction. ESI-MS revealed up heavy-atom binding sites determined by $\mathrm{X}$-ray diffraction analysis (Sun \& Hammer, 2000). Of the two $\mathrm{KAu}(\mathrm{CN})_{2}$ reactions, the reaction with the $28: 1$ molar ratio of gold cyanide to native protein produced higher derivative-peak intensities than did the 9:1 molar ratio reaction, indicating a correlation between the mass-spectrometric peak intensity and the concentration of the heavy atom used in the deriva-

In short, mass spectrometry offers a rapid method for heavy-atom derivative screening. Compared with conventional screening by $\mathrm{X}$-ray diffraction, mass spectrometry can be used to screen potential derivatives in solution, thus eliminating the use of crystals. Typical heavy-atom derivatization reactions in solution and mass-spectrometric data acquisition can be completed in minutes to hours, compared with the days to weeks required for X-ray heavy-atom derivative data analysis. The limitation of this massspectrometry-based screening technique is that it has only been used for the detection of covalent adducts. It is not clear whether the method can be applied to noncovalently bound heavy atoms such as the lanthanides, although $\mathrm{Na}^{+}, \mathrm{Cl}^{-}$and other solvent ions are frequently detected as adducts to proteins in mass spectrometry.

\section{Derivatization by the quick-soak method}

Once heavy-atom compounds with good reactivities in the crystallization buffer have been identified and their ability to react with the protein of interest has been confirmed by mass spectrometry, the process of carrying out heavy-atom soaks with crystals begins. In order to streamline this process and reduce the changes in crystals during the soaking procedure, we have developed a quicksoak method. This method is generally less damaging to the crystals and tends to produce more isomorphous crystals and thus better phasing statistics than conventional soaking techniques. Massspectrometric measurements show that adducts of many covalent heavy-atom compounds are formed within minutes 
Table 4

Derivatization conditions and phasing statistics of Fc $\gamma$ RIII derivatives (adapted from Sun et al., 2002).

\begin{tabular}{lccccc}
\hline \multicolumn{3}{c}{ TMLA } & \multicolumn{3}{c}{$\mathrm{HgCl}_{2}$} \\
\hline Derivatization $\dagger$ & $\begin{array}{c}5 \mathrm{~m} M, \\
10 \mathrm{~min}\end{array}$ & $\begin{array}{c}10 \mathrm{~m} M, \\
10 \mathrm{~min}\end{array}$ & $\begin{array}{c}10 \mathrm{~m} M, \\
24 \mathrm{~h}\end{array}$ & $\begin{array}{c}\text { Saturated, } \\
10 \mathrm{~min}\end{array}$ & $\begin{array}{c}\text { Saturated, } \\
2 \mathrm{~h}\end{array}$ \\
$R_{\text {iso }}$ & 0.093 & 0.09 & 0.168 & 0.119 & 0.273 \\
Heavy-atom peak height $\neq$ & & & & \\
Site 1 $(\sigma)$ & 6.7 & 17.8 & $<5.0$ & 7.6 & 24.4 \\
Site 2 $(\sigma)$ & 6.0 & 12.8 & $<5.0$ & 5.2 & 16.4 \\
\hline
\end{tabular}

$\dagger$ Heavy-atom soaking concentration, soaking time. $\$$ The heavy-atom sites are shown as peak heights in standard deviations from the difference Fourier $\left(F_{\mathrm{PH}}-F_{\mathrm{P}}\right)$ map. The coordinates of sites 1 and 2 of the TMLA derivatives are $(111.99,12.54,13.41)$ and $(88.49$, $21.42,23.78) \AA$, respectively. The coordinates of sites 1 and 2 of the $\mathrm{HgCl}_{2}$ derivatives are $(80.27,1.80,27.71)$ and $(104.52,7.78,29.52) \AA$, respectively

in solution (Agniswamy et al., 2008) and this rapid reaction rate presumably also occurs within crystals. In the following section, we present a comparison of quick-soak-derived phasing statistics with those obtained using conventional longer soaks for crystals of a number of test cases including lysozyme, Fc $\gamma$ RIII, the extracellular domain of a type II human transforming growth factor $\beta$ (TGF- $\beta$ ) receptor (T $\beta$ RII) and the natural killer cell receptor NKG2D in complex with its ligand ULBP3.

\subsection{Derivatization of hen egg-white lysozyme crystals}

Two compounds previously known to derivatize lysozyme, $\mathrm{KAuCl}_{4}$ and $\mathrm{K}_{2} \mathrm{PtCl}_{6}$, were chosen to identify the optimal time for crystal soaking and the optimal heavy-metal concentrations that should be used. Both of the original derivatives were obtained after 7-14 d of soaking in the heavy-atom solution (Blake et al., 1974). For the quick-soak method, the lysozyme crystals were soaked in a $10 \mathrm{~m} M$ solution of heavy-atom compound for $10 \mathrm{~min}$, designated hereafter as the $(10 \mathrm{mM}$, $10 \mathrm{~min}$ ) soak. The data for $\mathrm{KAuCl}_{4}$ derivatives were collected from crystals using three different soaking conditions: (10 mM, $10 \mathrm{~min}),(10 \mathrm{~m} M, 24 \mathrm{~h})$ and $(1 \mathrm{~m} M, 48 \mathrm{~h})$ (Table 3$)$. Only the $10 \mathrm{~min}$ soak produced diffraction data that were similar in quality to the native data as judged by diffraction resolution, $R_{\text {merge }}$ and $I / \sigma(I)$ for the outermost resolution shell of reflections. Both the 24 and $48 \mathrm{~h}$ soaked crystals diffracted to lower resolution than did the native crystal. Interestingly, while the 10 min soaks resulted in the smallest isomorphous $R$ factors $\left(R_{\text {iso }}\right)$, the heavy-atom occupancies were the highest. Similar results were observed with the 10 min $\mathrm{K}_{2} \mathrm{PtCl}_{6}$ soak, which resulted in no reduction in the diffraction resolution of the lysozyme crystal, whereas once again the 22 and 48 h soaks resulted in weaker diffraction and lower heavy-atom occupancies (Table 3 ). When the data from three 10 min soaks with

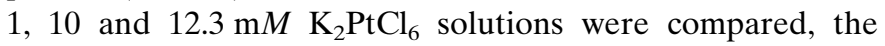
results showed significantly weaker binding of Pt in the $1 \mathrm{mM}$ soak compared with the 10 and $12.3 \mathrm{~m} M$ soaks. This suggests that the quick-soak method optimally requires a higher concentration of heavy-atom solution. While lengthy derivatization reactions ought to result in greater heavy-atom attachment, the observed lower heavy-atom occupancy associated with the longer soaks can be explained by a concomi- tant increase in non-isomorphism of the crystal arising from the longer soaking time. The lack of isomorphism can also be seen by the change in unit-cell parameters associated with the longer soaks, which is absent in the crystals soaked using the quick-soak procedure.

\subsection{Derivatives of Fc $\gamma$ RIII crystals}

Fc $\gamma$ RIII crystallized in space group $P 2{ }_{1} 2{ }_{1} 2$ and diffracted to $1.8 \AA$ resolution. Both trimethyllead acetate (TMLA) and $\mathrm{HgCl}_{2}$ reacted with the receptor as shown by mass spectrometry. Diffraction data were collected from three TMLAderivatization soaks: $(5 \mathrm{~m} M, 10 \mathrm{~min}),(10 \mathrm{~m} M, 10 \mathrm{~min})$ and $(10 \mathrm{~m} M, 24 \mathrm{~h})$. Similar to the lysozyme tests, the $(10 \mathrm{~m} M$, $10 \mathrm{~min}$ ) soak resulted in better heavy-atom derivatization than the 24 h soak (Table 4). A comparison between the two $10 \mathrm{~min}$ soaks with 5 and $10 \mathrm{~m} M$ TMLA showed that the lead occupancies in the $(10 \mathrm{~m} M, 10 \mathrm{~min})$ soak are more than twofold higher than those in the $(5 \mathrm{~m} M, 10 \mathrm{~min})$ soak, again indicating that the higher concentration of heavy-atom solution has a direct effect on derivatization. For $\mathrm{HgCl}_{2}$ soaking, Fc $\gamma$ RIII crystals were soaked in saturated $\mathrm{HgCl}_{2}$ (less than $5 \mathrm{mM}$ ) solution for different periods of time. Overnight soaks led to crystal lattice disorder and loss of diffraction. While both the $10 \mathrm{~min}$ and $2 \mathrm{~h}$ soaks resulted in $\mathrm{Hg}$ derivatization (Table 4), the two major $\mathrm{Hg}$-binding sites in the $2 \mathrm{~h}$ soak have higher occupancies than those obtained from the $10 \mathrm{~min}$ soak, suggesting that complete $\mathrm{HgCl}_{2}$ derivatization took longer than the TMLA-derivatization reaction and that the optimal

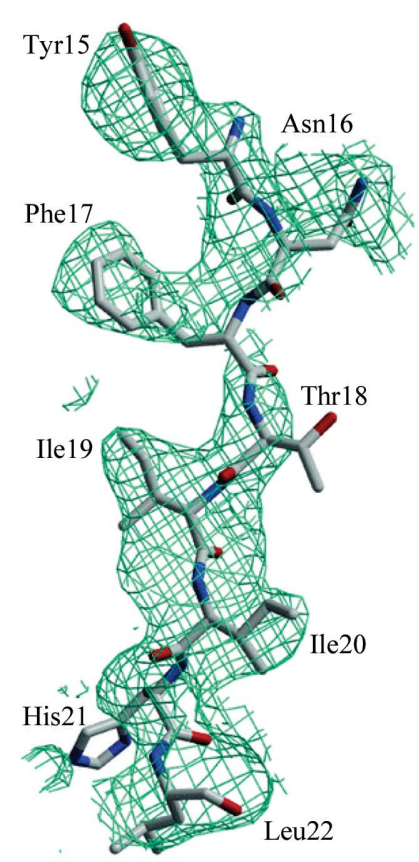

(a)

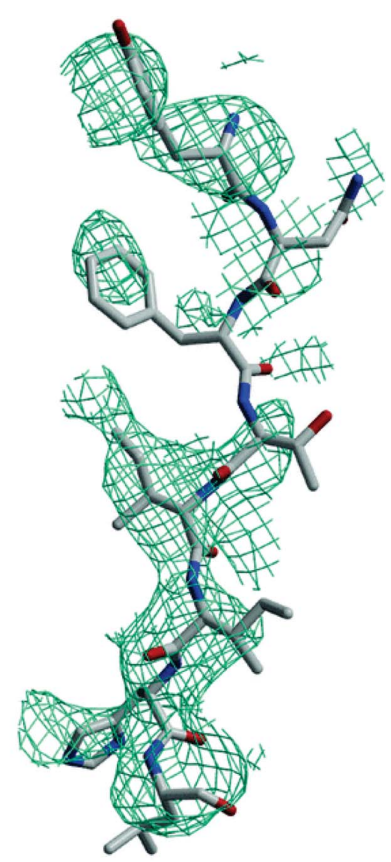

(b)
Figure 4

Experimental electron-density maps of the NKG2D-ULPB3 complex phased with a quick-soaked $\mathrm{K}_{2} \mathrm{PtCl}_{4}$ derivative. (a) Electron-density map generated from combined MAD and SIR phases contoured at $1 \sigma$ displaying a $\beta$-strand of ULBP3. (b) Electron-density map produced from MAD phases alone showing the same region as $(a)$ (taken from Sun \& Radaev, 2002). 
length of time for soaking may vary depending on the heavyatom compound and the protein under study.

\subsection{Phasing of the T/RRII structure}

The extracellular domain of the type II transforming growth factor- $\beta$ (TGF- $\beta$ ) receptor (T $\beta$ RII) has been expressed and crystallized (Boesen et al., 2000). Using mass spectrometry, $\mathrm{HgCl}_{2}$ was shown to derivatize T $\beta \mathrm{RII}$ in solution. Crystals of $\mathrm{T} \beta \mathrm{RII}$ were derivatized by soaking with saturated $\mathrm{HgCl}_{2}$ solution for $10 \mathrm{~min}$ and diffraction data were collected around the $\mathrm{Hg} L_{\mathrm{III}}$ absorption edge for structure determination using MAD. For comparison, equivalent MAD data sets were also collected from a crystal derivatized for $12 \mathrm{~h}$ using a heavyatom soaking solution identical to that used in the quick-soak experiment. Overall, the phasing statistics are very similar for

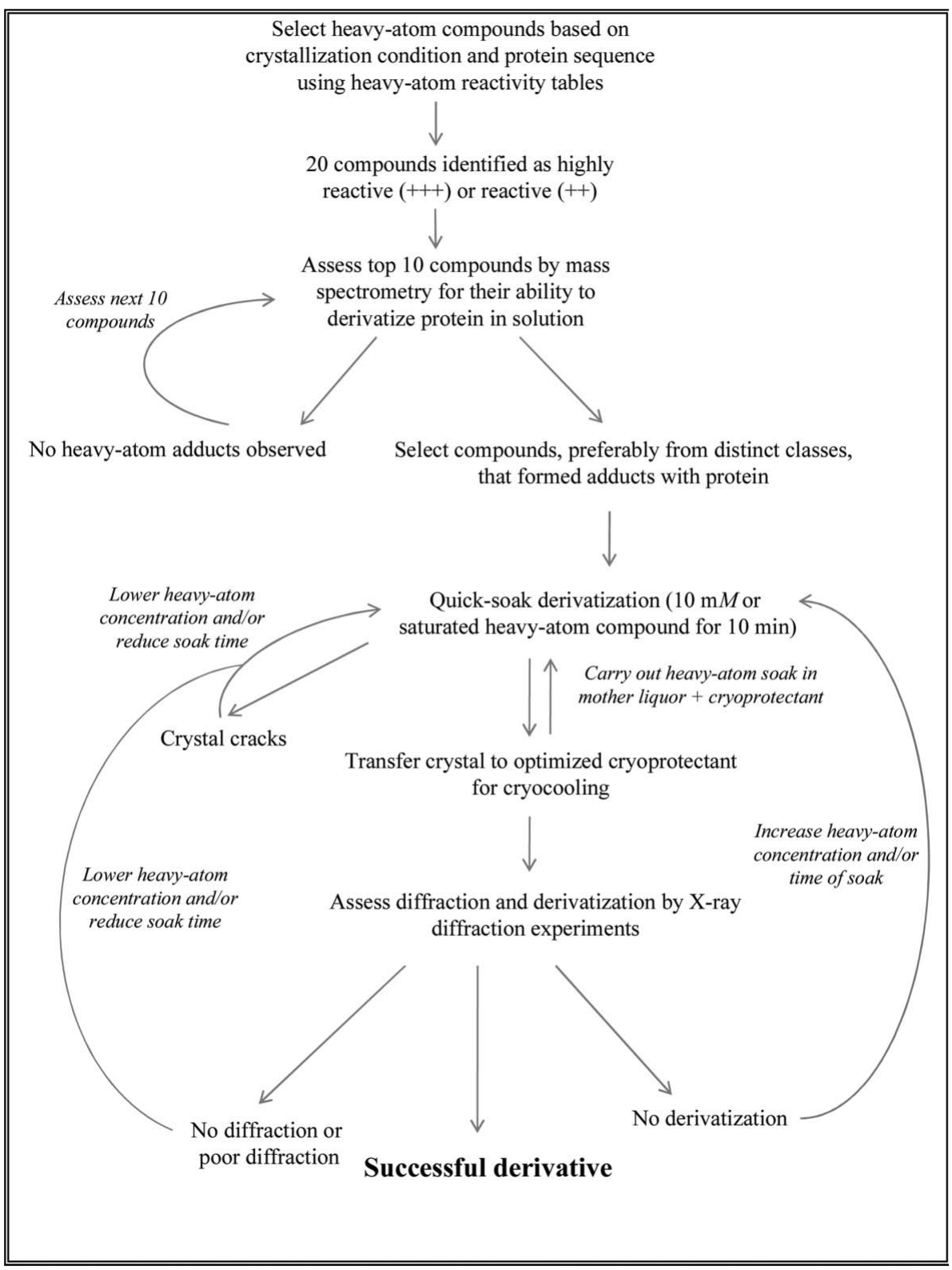

Figure 5

A flow chart outlining the major steps in the rational approach for heavy-atom derivative screening. both the quick-soak and the $12 \mathrm{~h}$ soak, illustrating the effectiveness of the quick-soak in derivatization and subsequent phasing. Again, the calculated $R_{\text {iso }}$ of the quick-soak derivative (0.23) is lower than that of the longer soak (0.37), indicating increased crystal non-isomorphism as a result of prolonged soaking. This is also reflected in a $1.1 \AA$ change in the unit-cell parameter $a$ in the case of the crystal soaked for $12 \mathrm{~h}$ compared with a $0.5 \AA$ change in $a$ for the crystal soaked for $10 \mathrm{~min}$. Since phases derived from isomorphous replacement $\left(F_{\mathrm{PH}}-F_{\mathrm{P}}\right)$ terms are affected by non-isomorphism between a derivative data set and a native data set, they are often inconsistent with phases derived from anomalous and multi-wavelength components. Attempts to combine these phases often yield electron-density maps that are poorer in quality than those calculated from MAD phasing alone. In this example, the combined phases (SIRAS map) from the shorter soak are not only better than those obtained from the longer soak but they are also better than the MAD phased map, clearly demonstrating the benefits of a quick-soak in reducing crystal nonisomorphism (Fig. 3).

\subsection{Phasing of the NKG2D-ULBP3 crystal}

NKG2D is a $14 \mathrm{kDa}$ C-type lectinlike receptor expressed on the surface of natural killer cells and certain $T$ cells. ULBP3 is a $24 \mathrm{kDa}$ class I major histocompatibility complex antigen-like molecule and a ligand of NKG2D. The crystals of the NKG2D-ULBP3 complex diffracted to $2.6 \AA$ resolution (Radaev et al., 2001). $\mathrm{K}_{2} \mathrm{PtCl}_{4}, \mathrm{KAuBr}_{4}$ and $\mathrm{KAuCl}_{4}$ showed heavy-atom adducts in mass-spectrometric analysis. Attempts to soak NKG2D-ULBP3 crystals for $24 \mathrm{~h}$ in solutions containing $1 \mathrm{~m} M$ of these heavy-atom compounds all resulted in lattice disorder and loss of diffraction beyond $6 \AA$ resolution. In contrast, a quick-soak of the crystals in $10 \mathrm{mM} \mathrm{K} \mathrm{K}_{2} \mathrm{PtCl}_{4}$ for $10 \mathrm{~min}$ resulted in no visual deterioration of the diffraction. A total of four Pt heavy-atom sites were determined and heavy-atom phasing resulted in an overall figure of merit of 0.41. Again, the combined SIR and MAD phases resulted in a better electron-density map than that calculated from the MAD phases alone (Fig. 4). It is worth emphasizing that only the quick-soak procedure resulted in a usable phasing derivative in this case and that all the longer soaks resulted in large crystal lattice disorder. 
Table 5

Rational heavy-atom screening of lysozyme (adapted from Agniswamy et al., 2008).

The extent of heavy-atom reactivity was evaluated based on the peak heights of observed derivatives from mass-spectrometric experiments and was assigned on a four-level scale as either,,-+++ or +++ , which equate to no significant derivative adduct formation and derivative adducts with peak heights less than $25 \%$, between 25 and $50 \%$ and above $50 \%$ of the native peak intensity, respectively.

\begin{tabular}{|c|c|c|}
\hline Compound & Peptide reactivity & Lysozyme reactivity \\
\hline MHTS & ND & Blake et al. (1962) \\
\hline $\mathrm{K}_{2} \mathrm{PdCl}_{4}$ & ND & Blake et al. (1962) \\
\hline $\mathrm{K}_{2} \mathrm{HgBr}_{4}$ & ND & Blake (1968) \\
\hline $\mathrm{K}_{2} \mathrm{HgI}_{4}$ & - & Blake et al. (1962) \\
\hline PCMB & - & Blake (1968) \\
\hline PCMBS & + & Blake (1968) \\
\hline $\mathrm{K}_{2} \mathrm{PtCl}_{6}$ & + & Blake (1968) \\
\hline $\mathrm{K}_{2} \mathrm{AuCl}_{4}$ & ++ & Blake (1968) \\
\hline $\mathrm{K}_{2} \mathrm{PtBr}_{4}$ & +++ & $+;$ Blake (1968) \\
\hline $\mathrm{K}_{2} \mathrm{PtCl}_{4}$ & +++ & +++; Blake (1968) \\
\hline $\mathrm{K}_{2} \mathrm{PtBr}_{6}$ & +++ & +++ \\
\hline Methylmercury(II) acetate & +++ & +++ \\
\hline Ethylmercury phosphate & +++ & +++ \\
\hline Mercury(II) acetate & +++ & +++ \\
\hline TELA & +++ & + \\
\hline Lead nitrate & +++ & +++ \\
\hline Lead acetate & +++ & +++ \\
\hline Diaminoplatinum dinitrate & +++ & + \\
\hline Gold(II) chloride & +++ & + \\
\hline Thiomersal & +++ & - \\
\hline Mersalyl & +++ & - \\
\hline Mercury(II) bromide & +++ & - \\
\hline Methylmercury(II) chloride & +++ & - \\
\hline Mercury(II) iodide & - & - \\
\hline Methylmercury(II) bromide & - & - \\
\hline $\mathrm{K}_{2} \mathrm{Pt}(\mathrm{CN})_{4}$ & - & +++ \\
\hline $\mathrm{K}_{2} \mathrm{PtI}_{6}$ & - & - \\
\hline Gold sodium thiosulfate & - & - \\
\hline Hexaphenyllead & - & - \\
\hline
\end{tabular}

Thus, the brief soaks are highly advantageous compared with conventional longer soaks for low-resolution diffracting crystals that could easily be damaged by heavy-atom soaks.

Compared with longer conventional soaks, the quick-soak method offers three main advantages. Firstly, it generally preserves the diffraction resolution of a crystal. In all examples tested, the quick-soak derivatization reactions resulted in no obvious deterioration of diffraction resolution compared with that of a native crystal. In contrast, data collected from overnight-soaked crystals often showed a reduction in both resolution and data quality. In some cases, the longer overnight soaks resulted in complete lattice disorder. Secondly, the quick-soak method minimizes the non-isomorphism associated with a derivative data set. This is reflected in smaller unit-cell parameter changes and better phasing statistics in all the quick-soak examples described here. Thirdly, the quicksoak method saves time and offers the potential for highthroughput 'on-the-fly' real-time heavy-atom screening.

\subsection{Choice of heavy-atom concentration and soaking time}

In conventional soaks, the concentration of a heavy-atom reagent is often limited by its adverse effects on the crystal lattice and subsequently the diffraction resolution. These adverse effects are negligible in all four quick-soak test cases described above. Consequently, for the benefit of thorough derivatization, a higher concentration of heavy-atom reagents can and should be used in quick-soak experiments. In both the lysozyme and Fc $\gamma$ RIII examples the highest heavy-atom occupancies were obtained with a $10 \mathrm{~m} M$ or higher concentrations of the heavy-atom reagent. Most of the quick-soak experiments were carried out for time periods between $10 \mathrm{~min}$ and $2 \mathrm{~h}$. The optimum soaking time is a balance between achieving high heavyatom binding occupancy and minimizing crystal non-isomorphism arising from the soaking procedure.

\section{Applying rational heavy-atom screening to lysozyme}

The rational heavy-atom screening strategy is summarized in a flow chart (Fig. 5). As a test case, we applied this rational approach to lysozyme in order to illustrate the gains that can be achieved using this strategy.

Under the crystallization conditions of hen egg-white lysozyme, 15 heavyatom compounds are predicted to be highly reactive based on the lysozyme amino-acid sequence (Table 5). Only two of these 15 compounds, $\mathrm{K}_{2} \mathrm{PtCl}_{4}$ and $\mathrm{K}_{2} \mathrm{PtBr}_{4}$, overlap with those phasing derivatives used by Blake (1968) in the initial structure determination. Several compounds known to derivatize lyso-
Figure 6

Difference Fourier $\left(F_{\mathrm{o}}-F_{\mathrm{c}}\right)$ maps calculated for lysozyme derivatized with lead acetate (blue density) and with potassium tetracyanoplatinate(II) (red density) and contoured at the $3 \sigma$ level. The structure of lysozyme is shown in ribbon representation, with the residues coordinating heavy atoms shown in ball-and-stick representation. PyMOL was used to generate the figure (taken from Agniswamy et al., 2008). 
zyme are not highly reactive with the model peptides in the lysozyme crystallization buffer, suggesting that they may not be optimal for phasing. These 15 heavy-atom compounds were assessed by mass spectrometry to confirm their reactivity with lysozyme. Except for four mercury compounds that were selected based on their reactivities with the cysteine peptide, the remaining 11 compounds all reacted with lysozyme in solution (Table 5). The failure of the four mercury compounds to derivatize lysozyme is likely to be a consequence of the lack of freely accessible cysteines in the protein. When a protein contains free cysteines they can be highly reactive with many heavy-atom compounds and thus may play a critical role in successful derivatization. In addition, six compounds which failed to react with the peptides in the sodium acetate buffers were selected for test reactions with lysozyme in order to verify that these compounds are less reactive (Table 5). With the exception of $\mathrm{K}_{2} \mathrm{Pt}(\mathrm{CN})_{4}$, no adduct formation was observed between lysozyme and these test compounds.

Lead acetate, one of the compounds identified as highly reactive in this study but not previously known to derivatize lysozyme, and $\mathrm{K}_{2} \mathrm{Pt}(\mathrm{CN})_{4}$ were used to soak lysozyme crystals using the quick-soak method. The soaked crystals were then analyzed to assess the quality of the data obtained and the extent of derivatization achieved. Three lead-binding sites were identified from the difference Fourier map (Fig. 6). In contrast, only a minor site was observed in the case of the $\mathrm{K}_{2} \mathrm{Pt}(\mathrm{CN})_{4}$-derivatized crystal. All three lead-binding sites exhibited higher occupancy than the platinum site and the lead derivative also had a higher figure of merit, indicating its potential as a phasing derivative (Table 6). The results show that while compounds which failed to react with the model peptides may still derivatize a protein in solution, they are likely to produce only minor binding sites in the crystal structure.

\section{Conclusion}

In summary, it is possible to streamline the conventional heavy-atom derivatization procedure. Use of heavy-atom reactivity profiles allows the rational selection of potential heavy-atom compounds that are amenable to derivatization under experimental crystal-growth conditions. These potential candidates can then be evaluated for their ability to derivatize the target protein by mass spectrometry. In principle, both heavy-atom concentration and soaking time can be optimized using mass spectrometry. Upon verification by mass spectrometry in solution, derivatization reactions in crystals can be carried out using the quick-soak method to minimize nonisomorphism between native and derivatized crystals and thus
Table 6

Phasing statistics of heavy-atom derivatization of lysozyme using two test compounds (adapted from Agniswamy et al., 2008).

Values in parentheses are for the highest resolution shell.

\begin{tabular}{lll}
\hline & Lead acetate & $\mathrm{K}_{2} \operatorname{Pt}(\mathrm{CN})_{4}$ \\
\hline Unit-cell parameters $(\AA)$ & & \\
$\quad a$ & 78.967 & 77.977 \\
$b$ & 78.967 & 77.977 \\
$\quad c$ & 37.104 & 36.983 \\
Resolution $(\AA)$ & $50-1.84(1.91-1.84)$ & $50-2.5(2.59-2.5)$ \\
Completeness $(\%)$ & $97.4(94.3)$ & $87.5(91.6)$ \\
$R_{\text {merge }}$ & $0.051(0.165)$ & $0.11(0.362)$ \\
$I / \sigma(I)$ & $29.46(9.77)$ & $11.41(3.24)$ \\
$R_{\text {iso }}$ & 0.109 & 0.319 \\
Figure of merit & 0.235 & 0.144 \\
Heavy-atom peak height $($ in $\sigma)$ & & \\
$\quad$ Site 1 & 14.6 & 4.91 \\
Site 2 & 10.92 & N/A \\
Site 3 & 5.16 & N/A \\
\hline
\end{tabular}

improve phasing. Overall, the method replaces the most laborious and time-consuming steps in conventional heavyatom derivatizations with a prediction-based rational approach that should increase the likelihood of successful derivatization and maximize the quality of heavy-atom phases.

\section{References}

Agniswamy, J., Joyce, M. G., Hammer, C. H. \& Sun, P. D. (2008). Acta Cryst. D64, 354-367.

Blake, C. C. (1968). Adv. Protein Chem. 23, 59-120.

Blake, C. C., Fenn, R. H., North, A. C., Phillips, D. C. \& Poljak, R. J. (1962). Nature (London), 196, 1173-1176.

Blake, C. C., Geisow, M. J., Swan, I. D., Rerat, C. \& Rerat, B. (1974). J. Mol. Biol. 88, 1-12.

Blundell, T. L., Dodson, G. G., Dodson, E., Hodgkin, D. C. \& Vijayan, M. (1971). Recent Prog. Horm. Res. 27, 1-40.

Blundell, T. L. \& Jenkins, J. A. (1977). Chem. Soc. Rev. 6, 139-171.

Blundell, T. L. \& Johnson, L. N. (1976). Protein Crystallography, pp. 183-239. London: Academic Press.

Boesen, C. C., Motyka, S. A., Patamawenu, A. \& Sun, P. D. (2000). Protein Expr. Purif. 20, 98-104.

Boggon, T. J. \& Shapiro, L. (2000). Structure, 8, 143-149.

Garman, E. \& Murray, J. W. (2003). Acta Cryst. D59, 1903-1913.

Islam, S. A., Carvin, D., Sternberg, M. J. E. \& Blundell, T. L. (1998). Acta Cryst. D54, 1199-1206.

Radaev, S., Rostro, B., Brooks, A. G., Colonna, M. \& Sun, P. D. (2001). Immunity, 15, 1039-1049.

Rould, M. A. (1997). Methods Enzymol. 276, 461-472.

Snyder, G. A., Brooks, A. G. \& Sun, P. D. (1999). Proc. Natl Acad. Sci. USA, 96, 3864-3869.

Sun, P. D. \& Hammer, C. H. (2000). Acta Cryst. D56, 161-168.

Sun, P. D. \& Radaev, S. (2002). Acta Cryst. D58, 1099-1103.

Sun, P. D., Radaev, S. \& Kattah, M. (2002). Acta Cryst. D58, 10921098. 